\title{
Changes in the bony alignment of the foot after tendo-Achilles lengthening in patients with planovalgus deformity
}

\author{
Nak Tscheol Kim ${ }^{1 \dagger}$, Young Tae Lee ${ }^{1 \dagger}$, Moon Seok Park ${ }^{1,2}$, Kyoung Min Lee ${ }^{1,2}$, Oh Sang Kwon ${ }^{1}$ and \\ Ki Hyuk Sung ${ }^{1,2^{*}}$ (iD
}

\begin{abstract}
Background: This study was performed to investigate the change in the bony alignment of the foot after tendoAchilles lengthening (TAL) and the factors that affect these changes in patients with planovalgus foot deformity.

Methods: Consecutive 97 patients (150 feet; mean age 10 years; range 5.1-35.7) with Achilles tendon contracture (ATC) and planovalgus foot deformity who underwent TAL were included. All patients underwent preoperative and postoperative weight-bearing anteroposterior (AP) or lateral (LAT) foot radiographics. Changes in AP talo-1st metatarsal angle, AP talo-2nd metatarsal angle, LAT talo-1st metatarsal angle, and calcaneal pitch angle and the factors affecting such changes after TAL were analyzed using lineal mixed model.

Results: There were no significant change in AP talo-1st metatarsal angle and AP talo-2nd metatarsal angle after TAL in patients with cerebral palsy (CP) $(p=0.236$ and 0.212$)$. However, LAT talo-1st metatarsal angle and calcaneal pitch angle were significantly improved after TAL $\left(13.0^{\circ}, p<0.001\right.$ and $\left.4.5^{\circ}, p<0.001\right)$. Age was significantly associated with the change in LAT talo-1st metatarsal angle after TAL $(p=0.028)$. The changes in AP talo-1st metatarsal angle, AP talo-2nd metatarsal angle, and calcaneal pitch angle after TAL were not significantly associated with the diagnosis ( $p=0.879,0.903$, and 0.056 ). However, patients with CP showed more improvement in LAT talo1st metatarsal angle $\left(-5.0^{\circ}, p=0.034\right)$ than those with idiopathic cause.

Conclusion: This study showed that TAL can improve the bony alignment of the foot in patients with planovalgus and ATC. We recommend that physicians should consider this study's findings when planning operative treatment for such patients.
\end{abstract}

Keywords: Planovalgus foot deformity, Achilles tendon contracture, Tendo-Achilles lengthening, Bony alignment

\section{Background}

Planovalgus foot deformity is one of the common foot deformities that is characterized by hindfoot valgus, decreased medial longitudinal arch, and forefoot

\footnotetext{
* Correspondence: skh1219@naver.com

${ }^{+}$Nak Tscheol Kim and Young Tae Lee contributed equally to this work. 'Department of Orthopedic Surgery, Seoul National University Bundang Hospital, 82 Gumi-ro 173 Beon-gil, Bundang-Gu, Sungnam, Gyeonggi 13620, Republic of Korea

${ }^{2}$ Department of Orthopedic Surgery, Seoul National University College of Medicine, Seoul, Republic of Korea
}

abduction [1-5]. The cause of this deformity is unclear, and it is considered an idiopathic etiology [2, 3]. It is often associated with the Achilles tendon contracture (ATC), which causes pain and functional disability $[6,7]$. It is common for patients with cerebral palsy $(\mathrm{CP})$ to have planovalgus foot deformity accompanied by ATC [8].

A passive stretching exercise of the Achilles tendon with the heel in an inverted position is recommended for patients with ATC $[3,4]$. Botulinum toxin injection

(c) The Author(s). 2021 Open Access This article is licensed under a Creative Commons Attribution 4.0 International License, which permits use, sharing, adaptation, distribution and reproduction in any medium or format, as long as you give appropriate credit to the original author(s) and the source, provide a link to the Creative Commons licence, and indicate if changes were made. The images or other third party material in this article are included in the article's Creative Commons licence, unless indicated otherwise in a credit line to the material. If material is not included in the article's Creative Commons licence and your intended use is not permitted by statutory regulation or exceeds the permitted use, you will need to obtain permission directly from the copyright holder. To view a copy of this licence, visit http://creativecommons.org/licenses/by/4.0/. The Creative Commons Public Domain Dedication waiver (http://creativecommons.org/publicdomain/zero/1.0/) applies to the data made available in this article, unless otherwise stated in a credit line to the data. 
can also be considered, especially in CP patient [9]. If patients do not respond to conservative treatment, operative treatment is indicated. Various operative methods such as arthroereisis, lateral column lengthening osteotomy, calcaneal medial displacement osteotomy, calcaneal-cuboid-cuneiform osteotomy, and arthrodesis can be considered to treat planovalgus foot deformity $[3-5,8,10-14]$. If ATC is present, tendoAchilles lengthening (TAL) should be performed concomitantly with the planovalgus foot surgery [15-17].

Limited ankle dorsiflexion (ADF) due to ATC results in midfoot breakage and planovalgus foot deformity as ADF occurs at the mid-tarsal joint $[18,19]$. Thus, we think that planovalgus foot deformity may be improved after TAL in terms of radiographic parameters as improvement in dorsiflexion at ankle joint following TAL results in the improvement of midfoot breakage. However, there has been no evidence that isolated TAL changes the degree of planovalgus foot deformity. Therefore, we performed this study to investigate the changes in bony alignment of the foot after TAL and the affecting factors in patients with planovalgus foot deformity.

\section{Materials and methods}

This study was approved by Institutional Review Board at our hospital. The need for obtaining informed consent from the study subjects was waived due to the retrospective nature of our study.

Inclusion criteria were as follows: (1) consecutive patients who underwent TAL for ATC, (2) patients who had planovalgus foot deformity, and (3) patients who had preoperative and postoperative standing foot radiographs. Patients who underwent concomitant foot surgery for the correction of planovalgus foot deformity and those who did not have the relevant radiographs were excluded from this study. We reviewed the medical records and gathered data pertaining to patient demographics including age at surgery, sex, follow-up duration, diagnosis (cerebral palsy vs. idiopathic cause), and gross motor function classification system (GMFCS) level.

ATC was defined as ADF $<0^{\circ}$ with knee extension during physical examination. During measurement of $\mathrm{ADF}$, the foot was inverted to lock the mid-tarsal joint so as to avoid ADF at the mid-tarsal joint.

Table 1 Summary of patient demographics and clinical characteristics

\begin{tabular}{ll}
\hline Parameters & Values \\
\hline Sex (male/female) & $59 / 38$ \\
Age at surgery (years) & $10.0 \pm 5.9$ (range 5.1-35.7) \\
Body side (right/left) & $76 / 74$ \\
Etiology (cerebral palsy/idiopathic) & $89(135$ feet)/8 (15 feet) \\
\hline
\end{tabular}

Our clinical data warehouse included 237 patients with 387 feet. After implementation of the inclusion and exclusion criteria, 97 consecutive patients (150 feet) were finally enrolled in this study. The mean age at the time of surgery was 10.0 years (range 5.1-35.7), and mean follow-up duration was 2.7 years (range $0.5-10.5$ ). The etiology of patients was idiopathic in 8 patients (15 feet) and cerebral palsy in 89 patients (135 feet) (Table 1).

\section{Operative protocol}

TAL was performed using coronal Z-plasty, and the intraoperative goal was to achieve ankle dorsiflexion of $10^{\circ}$ in knee extension [20]. After surgery, all patients had to use a short leg cast and were

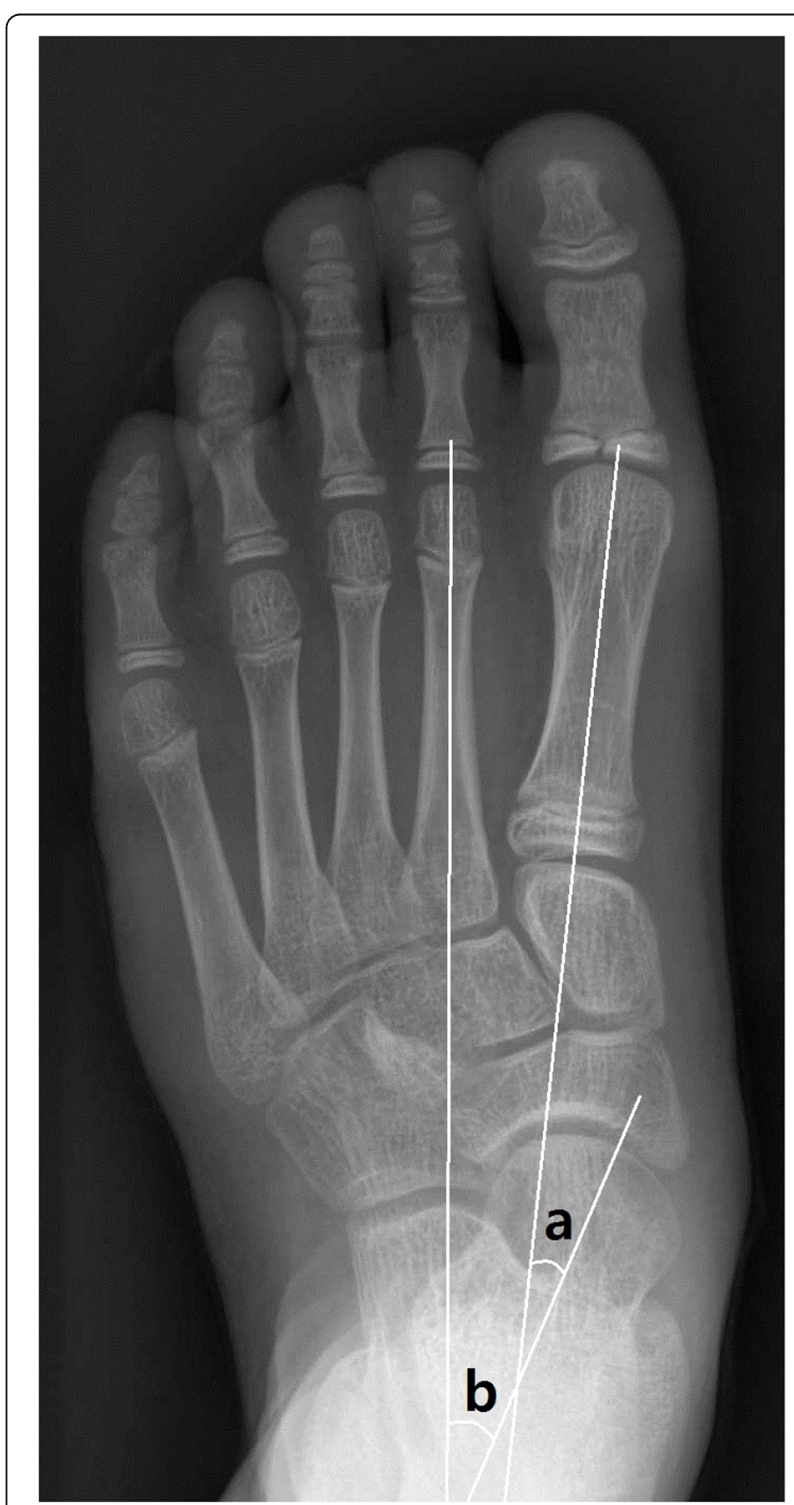

Fig. 1 On weight-bearing anteroposterior (AP) foot radiographs, AP talo-1st metatarsal angle (a) and AP talo-2nd metatarsal angle (b) were measured 


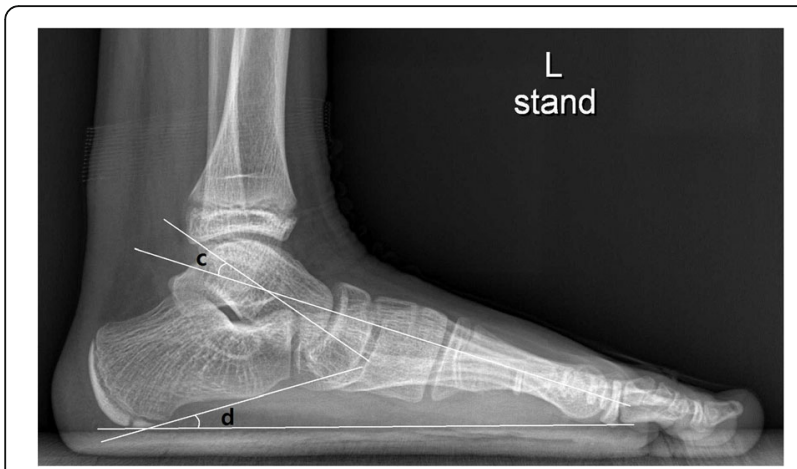

Fig. 2 On weight-bearing lateral (LAT) foot radiographs, LAT talo-1st metatarsal angle (c) and calcaneal pitch angle (d) were measured

recommended a non-weight-bearing period for 4 weeks. Thereafter, stretching, muscle-strengthening, and gait training were resumed with ankle foot orthosis, which was worn for 3 months.

\section{Acquisition of radiographic data}

The radiographic evaluation included assessment of standing anteroposterior (AP) and lateral (LAT) foot radiographs. The foot radiographs were taken using a UT 2000 X-ray machine (Philips Research, Eindhoven, The Netherlands) at a source-to-image distance of 100 $\mathrm{cm}$; the device was set to $50 \mathrm{kVp}$ and $5 \mathrm{mAs}$, and radiography was performed with the patients in the standing position. The radiographic images were retrieved using a picture archiving and communication system (PACS) (IMPAX; Agfa Healthcare, Mortsel, Belgium), and radiographic measurements were performed using the PACS software.

Four radiographic parameters on standing foot AP and LAT foot radiograph were selected to assess the degree of planovalgus foot deformity, AP talo-1st metatarsal angle, AP talo-2nd metatarsal angle, LAT talo-1st metatarsal angle, and calcaneal pitch angle [21-24]. On AP standing foot radiographs, AP talo-1st metatarsal angle was measured as the angle between a line drawn through the midpoints of the talar head and neck, and a line bisecting the long axis of the first metatarsal bone. AP talo-2nd metatarsal angle was measured as the angle between a line drawn through the midpoints of the talar head and neck, and a line bisecting the long axis of the second metatarsal bone (Fig. 1). On the LAT standing foot radiographs, LAT talo-1st metatarsal angle was measured as the angle between a line bisecting the long axis of the first metatarsal bone and a line drawn through the midpoints of the talar head and neck. Calcaneal pitch angle was measured as the angle between a line drawn through inferior border of the 5 th metatarsal head and calcaneal tuberosity, and a line drawn along the lower margin of the calcaneus (Fig. 2).

Before performing the main measurements, we performed reliability testing for radiographic measurements. Sample size estimation showed that a minimum of 36 feet (18 left and 18 right) radiographs should be assessed. Three orthopedic surgeons with 3,8 , and 8 years' experience, respectively in independent practice, measured the radiographs in a blinded fashion and determined interobserver reliability using intraclass correlation coefficients (ICCs) and 95\% confidence interval.

\section{Statistical analyses}

Reliability was assessed by the ICC in the setting of a 2 -way mixed-effect model, assuming a single measurement and absolute agreement [25]. With a target ICC value of 0.8 and $95 \% \mathrm{CI}$ width of 0.2 for 3 examiners, the minimal sample size was 36 feet using Bonett's methods [26].

Descriptive statistics were used to summarize the patients' demographics and radiographic measurements. Comparison between preoperative and postoperative radiographic measurements was performed using a paired $t$-test. For the purpose of statistical independence, linear mixed model (LMN) was used for statistical analysis. To estimate the extent of change of radiographic measurements after TAL, multiple factors were adjusted using a LMN with age, sex, diagnosis, and GMFCS as the fixed effects and individual subjects as the random effects.

Table 2 Changes in radiographic measurements after tendo-Achilles lengthening

\begin{tabular}{|c|c|c|c|c|c|c|c|c|c|c|}
\hline \multirow[t]{2}{*}{ Parameters $\left({ }^{\circ}\right)$} & \multicolumn{2}{|l|}{ Total } & \multirow[b]{2}{*}{$p$} & \multicolumn{3}{|l|}{ Cerebral palsy } & \multicolumn{3}{|l|}{ Idiopathic } & \multirow{2}{*}{$\begin{array}{l}p \\
\text { (between } \\
\text { group) }\end{array}$} \\
\hline & Preoperative & Postoperative & & Preoperative & Postoperative & $p$ & Preoperative & Postoperative & $p$ & \\
\hline $\begin{array}{l}\text { AP talus-1st meta } \\
\text { tarsal angle }\end{array}$ & $14.8 \pm 8.1$ & $11.9 \pm 10.5$ & 0.017 & $14.8 \pm 8.9$ & $11.8 \pm 11.4$ & 0.054 & $14.9 \pm 5.0$ & $12.1 \pm 6.7$ & 0.045 & 0.190 \\
\hline $\begin{array}{l}\text { AP talus-2nd meta } \\
\text { tarsal angle }\end{array}$ & $24.5 \pm 8.1$ & $20.4 \pm 11.7$ & 0.006 & $24.0 \pm 8.8$ & $19.8 \pm 12.7$ & 0.026 & $26.1 \pm 4.8$ & $22.4 \pm 7.1$ & 0.011 & 0.444 \\
\hline $\begin{array}{l}\text { Lateral talus-1st } \\
\text { metatarsal angle }\end{array}$ & $21.5 \pm 10.8$ & $12.2 \pm 10.2$ & $\begin{array}{l}< \\
0.001\end{array}$ & $22.2 \pm 10.8$ & $12.4 \pm 10.3$ & $\begin{array}{l}< \\
0.001\end{array}$ & $15.0 \pm 8.6$ & $10.3 \pm 8.7$ & 0.001 & 0.022 \\
\hline $\begin{array}{l}\text { Calcaneal pitch } \\
\text { angle }\end{array}$ & $9.3 \pm 7.0$ & $13.3 \pm 6.2$ & $\begin{array}{l}< \\
0.001\end{array}$ & $8.4 \pm 6.4$ & $12.6 \pm 5.8$ & $\begin{array}{l}< \\
0.001\end{array}$ & $17.0 \pm 7.4$ & $19.2 \pm 7.0$ & 0.003 & 0.021 \\
\hline
\end{tabular}


Table 3 Change of radiographic parameters and affecting factors after tendo-Achilles lengthening in patients with cerebral palsy

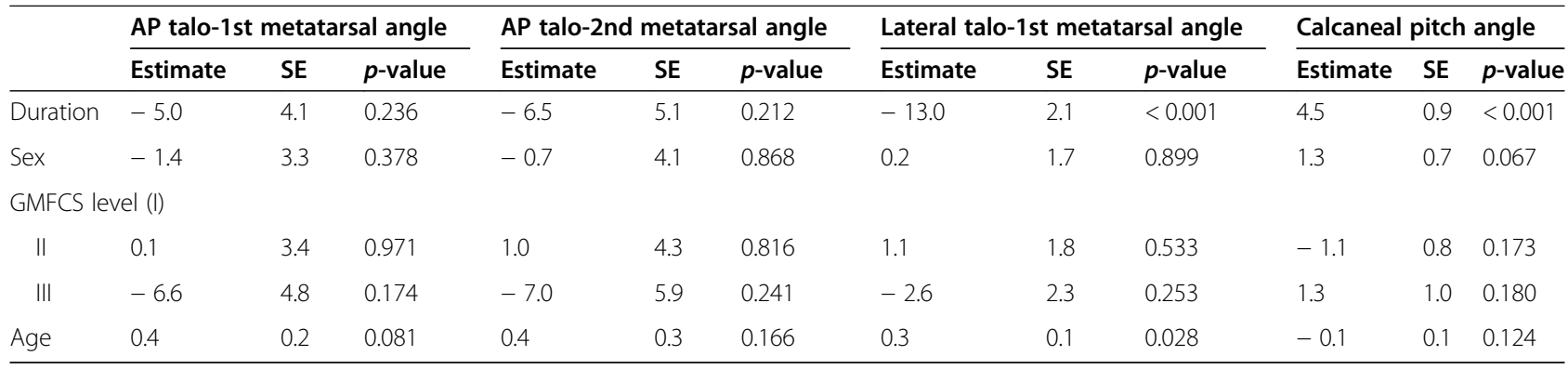

SE standard error

Statistical analyses were performed using the SPSS software for Windows (version 25.0; SPSS, Inc., Chicago, IL, USA) and R version 3.5.3 (R Foundation for Statistical Computing, Vienna, Austria). All statistics were 2tailed, and $p$-values $<0.05$ were considered significant.

\section{Results}

All radiographic measurement showed excellent interobserver reliabilities (ICC with 0.914 to 0.968 ). All radiographic parameters significantly improved after TAL (all $p<0.001$ ) (Table 2).

After adjusting for multiple factors, there were no significant change in AP talo-1st metatarsal angle and AP talo-2nd metatarsal angle after TAL in patients with $\mathrm{CP}$ ( $p=0.236$ and 0.212$)$. However, LAT talo-1st metatarsal angle and calcaneal pitch angle were significantly improved after TAL (all $p<0.001$ ). Age was significantly associated with the change in LAT talo-1st metatarsal angle after TAL $(p=0.028)$. Sex and GMFCS level were not associated with the change in radiographic parameters (Table 3).

Change in AP talo-1st metatarsal angle, AP talo-2nd metatarsal angle, and calcaneal pitch angle after TAL were not significantly associated with the diagnosis $(p=0.879$, 0.903 , and 0.056 , respectively). However, change in the LAT talo-1st metatarsal angle was significantly affected by the diagnosis $(p=0.034)$. Patients with cerebral palsy showed more improvement in LAT talo-1st metatarsal angle $\left(-5.0^{\circ}\right)$ than those with idiopathic cause (Table 4).

During the follow-up, calcaneal pitch angle significantly increased by $0.2^{\circ}$ per year $(p=0.017)$. However, there were no significant changes in AP talo-1st metatarsal angle, AP talo-2nd metatarsal angle, and LAT talo-1st metatarsal angle $(p=0.477,0.269$, and 0.076 , respectively).

\section{Discussion}

This study was performed to investigate the extent of radiographic changes in the foot and the factors affecting such as changes after TAL in patients with planovalgus foot deformity. This study found that two LAT radiographic parameters (LAT talo-1st metatarsal angle and calcaneal pitch angle) were considerably improved after TAL, and age and diagnosis were considerably associated with these changes.

There are some limitations to this study. First, the outcomes of TAL were assessed only by the radiographic parameters, although clinical outcomes such as foot pressure distribution and questionnaire are also important after surgery. Therefore, further study including clinical outcomes after TAL is needed. Second, although radiographic measurement of the hindfoot alignment view was important for evaluating hindfoot alignment, it was not performed due to retrospective design of this study. However, it has been reported that the radiographic parameters on standing foot AP and lateral radiographs were clinically relevant in terms of reliability, discriminant validity, and convergent validity [27]. Third, the follow-up period was not long enough to evaluate the long-term effects of TAL on radiographic foot alignment although this study's findings indicate no deterioration in terms of the radiographic parameters assessed. In order to investigate the long-term

Table 4 Change of radiographic parameters after tendo-Achilles lengthening according to diagnosis

\begin{tabular}{|c|c|c|c|c|c|c|c|c|c|c|c|c|}
\hline & \multicolumn{3}{|c|}{ AP talo- $1^{\text {st }}$ metatarsal angle } & \multicolumn{3}{|c|}{ AP talo- $2^{\text {nd }}$ metatarsal angle } & \multicolumn{3}{|c|}{ Lateral talo- $1^{\text {st }}$ metatarsal angle } & \multicolumn{3}{|c|}{ Calcaneal pitch angle } \\
\hline & Estimate & SE & $p$-value & Estimate & SE & $p$-value & Estimate & SE & $p$-value & Estimate & SE & $p$-value \\
\hline Duration & -3.3 & 3.6 & 0.363 & -3.5 & 4.5 & 0.441 & -7.8 & 2.8 & 0.006 & 2.4 & 1.2 & 0.051 \\
\hline Sex & -2.1 & 2.5 & 0.421 & -2.4 & 3.1 & 0.448 & 0.8 & 1.5 & 0.574 & 0.7 & 0.7 & 0.258 \\
\hline Diagnosis & -0.4 & 2.9 & 0.878 & -0.4 & 3.5 & 0.903 & -5.0 & 2.4 & 0.034 & 2.0 & 1.0 & 0.056 \\
\hline Age & 0.2 & 0.2 & 0.198 & 0.2 & 0.2 & 0.408 & 0.3 & 0.1 & 0.056 & -0.1 & 0.1 & 0.212 \\
\hline
\end{tabular}

SE standard error 


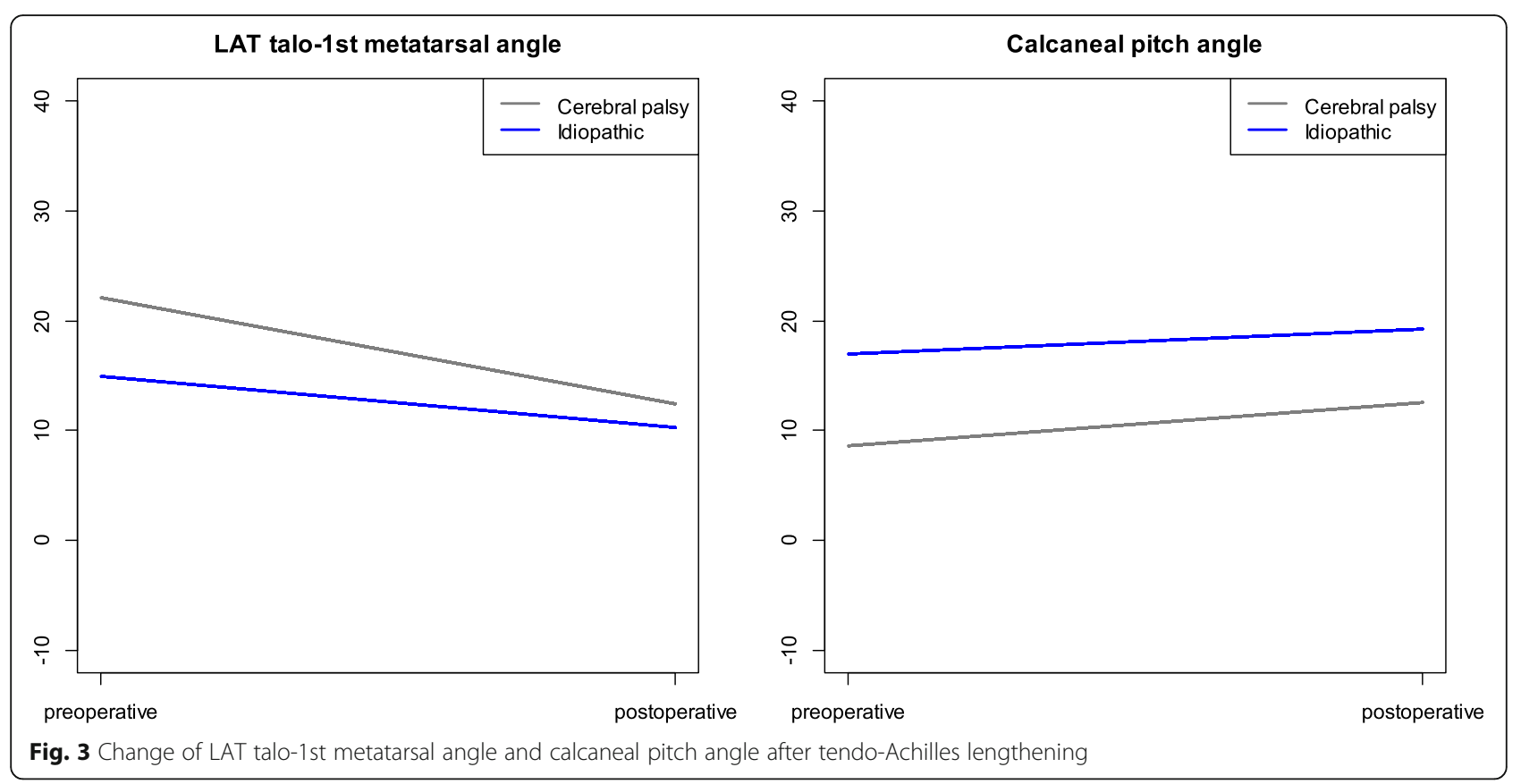

prognosis, additional long-term follow-up study is required.

If the $\mathrm{ADF}$ is restricted due to ATC, ADF occurs at the mid-tarsal joint, and this may eventually lead to midfoot breakage and planovalgus foot deformity [18, 19]. ATC can be treated by TAL, which enables ADF at the ankle joint. Therefore, TAL can result in the improvement of midfoot breakage and LAT radiographic parameters. LAT talo-1st metatarsal angle is the angle between the longitudinal axis of the first metatarsal bone and talus on a LAT weight-bearing foot radiograph. Increased LAT talo-1st metatarsal angle indicates a more vertically positioned talus, which represents increased midfoot breakage and increased dorsiflexion at the midtarsal joint. Calcaneal pitch angle represents the relative position of the calcaneus to the ground, and decreased calcaneal pitch angle means increased dorsiflexion at the mid-tarsal joint. Therefore, our result that LAT radiographic parameters were considerably improved after TAL is a reasonable finding. The degree of improvement of planovalgus foot deformity was limited despite the considerable changes in radiographic parameters after TAL. Accordingly, other operative procedure to correct planovalgus foot deformity, such as lateral column lengthening, calcaneo-stop, calcaneal-cuboid-cuneiform osteotomy, and calcaneal medial displacement osteotomy should be considered if the deformity is severe.

In this study, the diagnosis (cerebral palsy vs. idiopathic cause) was considerably associated with the change of LAT talo-1st metatarsal angle after TAL. LAT talo-1st metatarsal angle improved more (by $5^{\circ}$ ) in patients with cerebral palsy than in those with an idiopathic deformity (Fig. 3). We think that this finding might be attributed to the following factors. First, the sample size for patients with idiopathic cause was too small in this study (8 patients, 15 feet). Therefore, further research with large number of patients with idiopathic cause is needed. Second, the degree of equinus deformity may be severe in patients with cerebral palsy than in patients with idiopathic cause. Therefore, degree of TAL might affect the change in radiographic parameters.

This study also found that age was considerably associated with the change in LAT talo-1st metatarsal angle after TAL. As the age at the time of operation increases by 1 year, the change in LAT talo-1st metatarsal angle decreases by $0.3^{\circ}$. Therefore, we think that it is better not to delay TAL to prevent the aggravation of planovalgus foot deformity in patients with ATC and midfoot break.

\section{Conclusions}

In conclusion, this study showed that TAL can improve the bony alignment of the foot in patients with planovalgus foot deformity and ATC. We recommend that physicians should consider this study's findings when planning operative intervention for such patients.

\section{Abbreviations}

ATC: Achilles tendon contracture; CP: Cerebral palsy; TAL: Tendo-Achilles lengthening; GMFCS: Gross motor function classification system; AP: Anteroposterior; LAT: Lateral; ICC: Intraclass correlation coefficient; LMN: Linear mixed model; ADF: Ankle dorsiflexion

\section{Acknowledgements}

The authors thank the Division of Statistics in Medical Research Collaborating Center at Seoul National University Bundang Hospital for statistical analyses. 


\section{Authors' contributions}

KHS and MSP designed the study. YTL and OSK accumulated the data. MSP, KML, and KHS coordinated and supervised data collection. YTL and KHS analyzed the data. NTK and YTL drafted the manuscript. The authors read and approved the final manuscript.

\section{Funding}

This study was supported by the National Research Foundation of Korea(NRF) grant funded by the Korea government (MSIT) (No. NRF2019R1C1C1010352).

\section{Availability of data and materials}

The datasets used and/or analyzed during the current study available from the corresponding author on reasonable request.

\section{Ethics approval and consent to participate}

This retrospective study was approved by the institutional review board of our hospital (B-1910/568-111), and informed consent was waived owing to the retrospective nature of the study.

\section{Consent for publication}

Not applicable

\section{Competing interests}

The authors declare no competing interests.

Received: 12 January 2021 Accepted: 1 February 2021

Published online: 08 February 2021

\section{References}

1. Saragas NP, Ferrao PNF. Adult acquired flat foot deformity: the jointpreserving procedures in stage II tibialis posterior tendon dysfunction. South Afr Orthop J. 2016;15(1):41-50.

2. Carr JB 2nd, Yang S, Lather LA. Pediatric pes planus: a state-of-the-art review. Pediatrics. 2016;137(3):e20151230.

3. Dare DM, Dodwell ER. Pediatric flatfoot: cause, epidemiology, assessment, and treatment. Curr Opin Pediatr. 2014;26(1):93-100.

4. Lima TC, Volpon JB. Calcaneal lateral column lengthening osteotomy for symptomatic flexible flatfoot in children and adolescents: a systematic review. Rev Col Bras Cir. 2018;45(6):e1969.

5. Kim JR, Shin SJ, Wang SI, Kang SM. Comparison of lateral opening wedge calcaneal osteotomy and medial calcaneal sliding-opening wedge cuboidclosing wedge cuneiform osteotomy for correction of planovalgus foot deformity in children. J Foot Ankle Surg. 2013;52(2):162-6

6. Arangio G, Rogman A, Reed JF 3rd. Hindfoot alignment valgus moment arm increases in adult flatfoot with Achilles tendon contracture. Foot Ankle Int. 2009:30(11):1078-82.

7. Barouk LS. The effect of gastrocnemius tightness on the pathogenesis of juvenile hallux valgus: a preliminary study. Foot Ankle Clin. 2014; 19(4):807-22.

8. Luo CA, Kao HK, Lee WC, Yang WE, Chang CH. Limits of calcaneal lengthening for treating planovalgus foot deformity in children with cerebral palsy. Foot Ankle Int. 2017;38(8):863-9.

9. Preiss RA, Condie DN, Rowley DI, Graham HK. The effects of botulinum toxin (BTX-A) on spasticity of the lower limb and on gait in cerebral palsy. J Bone Joint Surg Br. 2003;85(7):943-8.

10. Kumar S, Sonanis SV. Lateral column lengthening for adolescent idiopathic pes planovalgus deformity - systematic review. J Orthop. 2017:14(4):571-6.

11. Zaw H, Calder JD. Operative management options for symptomatic flexible adult acquired flatfoot deformity: a review. Knee Surg Sports Traumatol Arthrosc. 2010;18(2):135-42.

12. Kadhim M, Holmes $L$ Jr, Miller F. Long-term outcome of planovalgus foot surgical correction in children with cerebral palsy. J Foot Ankle Surg. 2013 52(6):697-703

13. Mosca VS. Calcaneal lengthening for valgus deformity of the hindfoot. Results in children who had severe, symptomatic flatfoot and skewfoot. J Bone Joint Surg Am. 1995:77(4):500-12.

14. Rathjen KE, Mubarak SJ. Calcaneal-cuboid-cuneiform osteotomy for the correction of valgus foot deformities in children. J Pediatr Orthop. 1998; 18(6):775-82.
15. Solan MC, Carne A, Davies MS. Gastrocnemius shortening and heel pain. Foot Ankle Clin. 2014;19(4):719-38.

16. Meszaros A, Caudell G. The surgical management of equinus in the adult acquired flatfoot. Clin Podiatr Med Surg. 2007;24(4):667-85 viii.

17. Lin Y, Cao J, Zhang C, Yang L, Duan X. Modified percutaneous Achilles tendon lengthening by triple hemisection for Achilles tendon contracture. Biomed Res Int. 2019:2019:1491796.

18. Kim HY, Shin HS, Ko JH, Cha YH, Ahn JH, Hwang JY. Gait analysis of symptomatic flatfoot in children: an observational study. Clin Orthop Surg. 2017;9(3):363-73.

19. Maurer JD, Ward V, Mayson TA, Davies KR, Alvarez CM, Beauchamp RD, et al. Classification of midfoot break using multi-segment foot kinematics and pedobarography. Gait Posture. 2014;39(1):1-6.

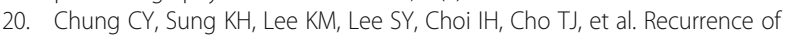
equinus foot deformity after tendo-achilles lengthening in patients with cerebral palsy. J Pediatr Orthop. 2015;35(4):419-25.

21. Westberry DE, Davids JR, Roush TF, Pugh LI. Qualitative versus quantitative radiographic analysis of foot deformities in children with hemiplegic cerebral palsy. J Pediatr Orthop. 2008;28(3):359-65.

22. Sung KH, Chung CY, Lee KM, Lee SY, Park MS. Calcaneal lengthening for planovalgus foot deformity in patients with cerebral palsy. Clin Orthop Relat Res. 2013:471(5):1682-90.

23. Sung KH, Kwon SS, Chung CY, Lee KM, Park MS. Radiographic changes of the mid-tarsal joint after calcaneal lengthening for planovalgus foot deformity. Foot Ankle Surg. 2020;26(1):110-5.

24. Davids JR, Gibson TW, Pugh LI. Quantitative segmental analysis of weightbearing radiographs of the foot and ankle for children: normal alignment. J Pediatr Orthop. 2005;25(6):769-76.

25. Lee KM, Lee J, Chung CY, Ahn S, Sung KH, Kim TW, et al. Pitfalls and important issues in testing reliability using intraclass correlation coefficients in orthopaedic research. Clin Orthop Surg. 2012;4(2):149-55.

26. Bonett DG. Sample size requirements for estimating intraclass correlations with desired precision. Stat Med. 2002:21(9):1331-5.

27. Lee KM, Chung CY, Park MS, Lee SH, Cho JH, Choi $I H$. Reliability and validity of radiographic measurements in hindfoot varus and valgus. J Bone Joint Surg Am. 2010;92(13):2319-27.

\section{Publisher's Note}

Springer Nature remains neutral with regard to jurisdictional claims in published maps and institutional affiliations.

Ready to submit your research? Choose BMC and benefit from:

- fast, convenient online submission

- thorough peer review by experienced researchers in your field

- rapid publication on acceptance

- support for research data, including large and complex data types

- gold Open Access which fosters wider collaboration and increased citations

- maximum visibility for your research: over $100 \mathrm{M}$ website views per year

At BMC, research is always in progress.

Learn more biomedcentral.com/submissions 